\title{
Gene therapy for neurodegenerative diseases: fact or fiction?
}

\author{
JANET E. CARTER and EDWARD H. SCHUCHMAN
}

Primary neurodegenerative diseases represent debilitating and progressive conditions for which no cure is currently available and for which therapeutic interventions remain limited to palliative and symptomatic treatment. Advances in gene transfer technology now present the realistic prospect of delivering therapeutic genes to the brain for neuroprotection, restoration of neuronal function or replacement of deficient proteins as treatments for these diseases. Thus, although central nervous system (CNS) gene therapy presents a number of unique challenges and clinical applications remain in their infancy, the realisation of this goal is approaching at an ever-increasing rate.

\section{THERAPEUTIC STRATEGIES - OVERVIEW OF APPROACHES}

Broadly speaking, four categories of therapeutic strategy have become available as a result of recent advances in genetics and cell biology. The first is gene therapy proper, which describes attempts to deliver 'healthy' genes to a target organ such as the brain. This category includes the specialised field of stem cell transplantation, which exploits the inherent biology of organ-specific stem cells to provide a source of genetically engineered replacement cells for the delivery of therapeutic genes to localised and widespread regions of the CNS. The second set of strategies aims to reduce expression of mutated genes and includes the use of antisense oligonucleotides and ribozymes. The third approach is to use positional cloning strategies to identify mutated genes involved in disease, and then to make use of the identified gene variants for new drug discovery. Finally, transplantation of non-genetically engineered primary embryonic neural tissue has been used in clinical trials, with varying degrees of success, to improve cognitive function and motor function in patients with Huntington's disease and Parkinson's disease, respectively. The first of these strategies (gene therapy) is discussed further here.

\section{GETTING GENES INTO CELLS - VECTOR-MEDIATED GENE DELIVERY}

To date, two approaches to gene delivery into somatic cells exist: ex vivo and in vivo. Ex vivo approaches require vectormediated gene transfer into cells before transplantation into the target, whereas in the context of the CNS in vivo refers to direct in situ intracerebral gene delivery. The vectors of choice for both methods of gene transfer are viruses modified to impair their replication after entering cells (i.e. replication-deficient). Five such viral vector systems are currently available, including retrovirus, adenovirus, adeno-associated virus, herpes simplex virus and lentivirus, together with a range of artificially constructed vectors or chimeras that are hybrids of the above.

All currently available viral vector systems have inherent limitations but it is important to note that innovations in vector design and purification strategies have helped to overcome some of these disadvantages. For example, 'gutless' adenoviral vectors, stripped of all structural viral genes and disguising pathogenic viruses in the 'coats' of non-virulent strains, help to minimise toxicity. Manipulation of specific gene sequences (i.e. promoters, enhancers) has been used to confine transgene expression to specific cell types in the brain and to produce longer-term expression (for a comprehensive review of viral vectors, see Hermans \& Verhaagen, 1998).

A number of non-viral gene transfer methods also have been developed for gene therapy, but at the present time the inefficiency of these methods precludes clinical application.

\section{CURRENT GENE THERAPY STRATEGIES FOR NEURODEGENER ATIVE DISEASES}

To date, gene therapy proper in the CNS has been tested only in experimental animal model systems and has included delivering genes encoding neurotrophic factors to inhibit neurodegeneration or stimulate regeneration, and the replacement of deficient neurotransmitter proteins or metabolic enzymes.

In an animal model of Parkinson's disease, for example, in vivo and ex vivo approaches to replace dopamine have included direct stereotactic intracerebral injection of viral vectors expressing the tyrosine hydroxylase gene to striatal neurons, or transplanting cells (fibroblasts or astrocytes) expressing tyrosine hydroxylase (Horrelou et al, 1994: Ridet et al, 1999). Co-expression of several enzymes on the biosynthetic pathway to dopamine (e.g. L-aromatic amino acid decarboxylase and tyrosine hydroxylase) from one viral vector or coincident expression of the two enzymes from two separate vectors, delivered at the same time, has also been used to maximise dopamine production. In a related strategy, tyrosine hydroxylase has been expressed virally together with guanosine5 '-triphosphate (GTP) cyclohydolase I, the enzyme necessary for generation of the tetrahydrobiopterin cofactor for tyrosine hydroxylase (Mandel et al, 1998). Although interpretation must remain cautious because of the limitations of Parkinsonian animal models, improvements in behaviour and increased dopamine synthesis have been reported. Neuroprotective paradigms for Parkinson's disease, in vivo and ex vivo, have included delivering genes encoding trophic factors such as glial-derived neurotrophic factor and brain-derived neurotrophic factor and anti-apoptotic genes such as the proto-oncogene Bcl-2 (Bilang-Bleuel et al, 1997; Choi-Lundberg et al, 1997). Encouraging results demonstrate successful rescue of degenerating striatal neurons and improved behavioural function in animal models.

Gene therapy in animal models for Huntington's disease has similarly concentrated upon protective ex vivo approaches, transplanting cells secreting nerve growth factor (NGF) or human ciliary neurotrophic factor (for a review, see Kordower et al, 1999). 
Experimental animal models with chemically induced lesions of cholinergic basal forebrain neurons have been used to mimic the behavioural and neurodegenerative changes of Alzheimer's disease. In ex vivo approaches, implantation of fibroblasts, genetically manipulated to produce NGF, into the basal forebrain before lesioning prevented degeneration of cholinergic neurons in an adult primate model (Tuszynski et al, 1996).

\section{STEM CELL \\ TRANSPLANTATION - SPECIALISED EX VIVO GENE THERAPY}

Perhaps one of the most remarkable advances for the field of CNS gene therapy has been the identification and isolation of neural stem cells (NSCs) from embryonic or foetal neural tissue. On transplantation into developing or adult mouse brain, human embryonic NSCs can migrate over distance, integrate into the surrounding microenvironment and demonstrate multipotency (i.e. an ability to differentiate into regionally appropriate neuronal cells); for a review, see Scheffler et al (1999). It is these inherent biological features that, on transplantation, allow for the generation of brain regions where integrated donorderived cells can replace lost cells, provide support to degenerating cells or, if genetically modified to do so, be used to target genes.

\section{IMMORTALISED STEM CELLS}

Neural stem cells also can be rendered 'immortal' by the addition of growth factors or by genetic manipulation, thereby promoting continuous cell division in vitro that is 'conditional' on the presence or absence of manipulatable factors such as temperature. Using this approach, immortalised NSCs from rodents, genetically modified in addition by viral vectors to express diseasespecific deficient enzymes or neurotrophic factors, have been used as biological 'minipumps' to correct the widespread neuropathology in animal models of lysosomal storage disease, mucopolysaccharidosis VII (Sly syndrome) and Tay-Sachs disease (Lacorazza et al, 1998). A similar approach has been employed to reconstitute partially the spatial memory deficits in rodent models of Alzheimer's disease generated by

JANET E. CARTER, MRCPsych, Department of Neuroscience and Section of Old Age Psychiatry, Institute of Psychiatry, King's College, London, UK and Department of Human Genetics, Mount Sinai School of Medicine, New York; EDWARD H. SCHUCHMAN, PhD, Department of Human Genetics, Mount Sinai School of Medicine, New York

Correspondence: Dr Janet Carter, Department of Neuroscience and Old Age Psychiatry, Institute of Psychiatry, De Crespigny Park, London SE5 8AF, UK. Tel: 0207848 0707; Fax: 02077080017 ; e-mail: janet.carter@iop.kcl.ac.uk

(First received 26 April 2000, final revision 27 October 2000, accepted II December 2000)

selective cholinergic lesioning (MartinezSerrano \& Bjorklund, 1998).

Significantly, homogeneous clonal populations of genetically immortalised human embryonic NSCs have been shown recently to be capable of differentiation into mature neuronal cell types on transplantation into adult rat striatum. This suggests their potential as vehicles for molecular therapies for neurodegenerative conditions (Rubio et al, 2000).

Despite the promise of brain-derived NSCs, there remain reservations about their use in transplantation to the mature CNS. It may be that the normal adult brain lacks the appropriate signals to realise their proliferative potential or that specific neuropathological conditions may alter the balance of required environmental signals. One answer may lie in exploiting the multipotency of stem cells by promoting lineage commitment in vitro from a founder stem cell population prior to transplantation to the damaged brain, to provide a limitless source of mature neuronal cells for transplantation and genetic manipulation.

\section{ALTERNATIVE STEM CELLS FOR EX VIVO CNS GENE THER APY}

The ongoing issues of limited accessibility and ethical concern over the use of foetal tissues have continued to drive the search for alternative populations of stem cells for neural transplantation. Stem cells derived from adult tissues such as the bone marrow and skin may have potential in this respect. A body of evidence undoubtedly now supports the extraordinary conclusion that adult stem cells extracted from their usual niche can be diverted from their normal fates and redirected to atypical lineages determined by their new microenvironment (Fuchs \& Segre, 2000). Remarkably, mesenchymal stem cells (mesodermal origin) isolated from human adult bone marrow demonstrate the ability to differentiate into astrocytic and neuronal phenotypes (neuroectodermal origin) when transplanted into the non-haematopoietic environment of the rodent brain - so-called inter-germ layer differentiation (Kopen $\mathrm{et} \mathrm{al}$, 1999; Pittenger et al, 1999). Because mesenchymal stem cells can be isolated readily from a patient's own bone marrow, their use in transplantation would circumvent the problem of graft versus host disease.

It can be seen that this radical transformation in the concepts of stem cell biology advances the prospect of achieving the ultimate goal for molecular medicine in the CNS: an easily isolated source of multipotent adult human stem cells with high proliferative capacity that can be lineagedirected into different types of neurons for the treatment of neurodegeneration.

\section{CONCLUSION}

Advances in gene transfer technologies now provide an array of versatile tools for gene delivery to localised or widespread areas of the brain. However, the optimism generated by results in animal models must be tempered because many improvements in safety, efficacy, stability and regulation of gene transfer are required before clinically effective CNS gene therapy can be accomplished for human patients. A cautionary note, too, is sounded by gene therapy attempts in organs with greater accessibility than the brain (e.g. liver, bone marrow), because these have, so far, met with disappointing outcomes. Despite such reservations, the successful application of these gene therapy strategies in treating CNS disorders in animal models already has demonstrated their enormous potential. Collaborative efforts between clinicians and basic science researchers continue to make CNS gene therapy an achievable goal. 


\section{ACKNOWLEDGEMENTS}

J.E.C. is a Medical Research Council Clinician Scientist Fellow. This article was supported in part by grants from the National Institutes of Health, including research grants $\mathrm{ROI} \mathrm{HD} 28607$ and $\mathrm{POI}$ HD28822.

\section{REFERENCES}

Bilang-Bleuel, A., Revah, F., Colin, P., et al (1997) Intrastriatal injection of an adenoviral vector expressing glial-cell-line derived neurotrophic factor prevents dopaminergic neuron degeneration and behavioural impairment in a rat model of Parkinson's disease. Proceedings of the National Academy of Sciences of the United States of America, 94, 8818-8823.

Choi-Lundberg, D. L., Lin, Q., Chang, Y., et al (1997) Dopaminergic neurones protected from degeneration by GDNF gene therapy. Science, 1997, 255, 838-84I.

Fuchs, E. \& Segre, J. A. (2000) Stem cells: a new lease on life. Cell, 100, 143-155.

Hermans, W. T. J. M. C. \& Verhaagen, J. (1998) Viral vectors, tools for gene transfer in the nervous system. Progress in Neurobiology, 55, 399-434.
Horrelou, P., Vigne, E., Castel, M., et al (1994) Direct intracerebral gene transfer of an adenoviral vector expressing tyrosine hydroxylase in a rat model of Parkinson's disease. Neuroreport, 6, 49-53.

Kopen, G. C., Prockop, D. J. \& Phinney, D. G. (1999) Marrow stromal cells migrate throughout forebrain and cerebellum and differentiate into astrocytes after injection into neonatal mouse brains. Proceedings of the National Academy of Sciences of the United States of America, 96, I07II-10716.

\section{Kordower, J., Isacson, O. \& Emerich, D. (1999)} Cellular delivery of trophic factors for the treatment of Huntington's disease. Experiments in Neurology, 159 4-20.

Lacorazza, H. F., Flax, J. D., Snyder, E. Y., et al (1998) Expression of human $\boldsymbol{\beta}$-hexosaminidase $\boldsymbol{\alpha}$-subunit gene (the gene defect of Tay-Sachs disease) in mouse brains upon the engraftment of transduced progenitor cells. Natural Medicine, 2, 424-429.

Mandel, R., Rendahl, K. G., Spratt, S. K., et al (1998) Characterisation of intrastriatal recombinant adenoassociated virus-mediated gene transfer of human tyrosine hydroxylase and human GTP-cyclohydrolase I in a rat model of Parkinson's disease. Journal of Neuroscience, 12, 427I-4284.
Martinez-Serrano, A. \& Bjorklund, A. (1998) Ex vivo nerve growth factor gene transfer to the basal forebrain in presymptomatic middle-aged rats prevents the development of cholinergic neuron atrophy and cognitive impairment during ageing. Proceedings of the National Academy of Sciences of the United States of America, 95, 1858-1863.

Pittenger, M. F., Mackay, A. M., Beck, S. C., et al (1999) Multilineage potential of adult mesenchymal stem cells. Science, 284, 143-147.

Ridet, J. L., Corti, O., Pencalet, P., et al (1999) Toward autologous ex vivo gene therapy for the central nervous system with human adult astrocytes. Human Gene Therapy, 20, 27I-280.

\section{Rubio, F. J., Bueno, C., Villa, A., et al (2000)}

Genetically perpetuated human neural stem cells engraft and differentiate into the adult mammalian brain. Molecular and Cell Neuroscience, 16, I-13.

Scheffler, B., Horn, M., Blumcke, L., et al (1999) Marrow-mindedness: a perspective on neuropoiesis. Trends in Neuroscience, 22, 348-357.

Tuszynski, M. H., Roberts, J., Senut, M. C., et al (1996) Gene therapy in the adult primate brain: intraparenchymal grafts of cells genetically modified to produce nerve growth factor prevent cholinergic neuronal degeneration. Gene Therapy, 4, 304-314. 